\title{
AUGUSTE, Claude Bonaparte et Marcel Bonaparte AUGUSTE, Les Déportés de Saint-Domingue. Contribution à l'histoire de l'Expédition française de Saint-Domingue (1802-1803). Préface de Robert Cornevin. Collection « Civilisations », no 10. Sherbrooke, Éditions Naaman, 1979. Ill., annexes, bibl., index des noms de personnes. 160 p. \$12.00.
}

\section{Jean-Marie Loncol}

Volume 36, numéro 1, juin 1982

URI : https://id.erudit.org/iderudit/304033ar

DOI : https://doi.org/10.7202/304033ar

Aller au sommaire du numéro

Éditeur(s)

Institut d'histoire de l'Amérique française

ISSN

0035-2357 (imprimé)

1492-1383 (numérique)

Découvrir la revue

Citer ce compte rendu

Loncol, J.-M. (1982). Compte rendu de [AUGUSTE, Claude Bonaparte et Marcel Bonaparte AUGUSTE, Les Déportés de Saint-Domingue. Contribution à l'histoire de l'Expédition française de Saint-Domingue (1802-1803). Préface de Robert Cornevin. Collection "Civilisations ", no 10. Sherbrooke, Éditions Naaman, 1979. Ill., annexes, bibl., index des noms de personnes. 160 p. \$12.00.] Revue d'histoire de l'Amérique française, 36(1), 107-109.

https://doi.org/10.7202/304033ar d'utilisation que vous pouvez consulter en ligne. 


\section{COMPTES RENDUS}

AUGUSTE, Claude Bonaparte et Marcel Bonaparte AUGUSTE. Les Déportés de Saint-Domingue. Contribution à l'histoire de l'Expédition française de Saint-Domingue (1802-1803). Préface de Robert Cornevin. Collection «Civilisations», no 10. Sherbrooke, Éditions Naaman, 1979. Ill., annexes, bibl., index des noms de personnes. 160 p. $\$ 12.00$

Membres en Côte d'Ivoire et au Canada de cette diaspora dont Haïti est la malheureuse victime, deux cousins ont établi entre Abidjan et Québec - où l'un travaillait comme médecin, l'autre comme professeur de relations internationales et d'économie politique - leur collaboration afin de publier ici une étude de la préface à l'émancipation de leur mère patrie que constitue l'Expédition française de 1802-1803.

Rappelons au lecteur pressé qu'Hispaniola, où Christophe Colomb implantait l'autorité de la Reine Catholique dès le 6 décembre 1492, avait d'abord vu tâtonner les commencements des Indes de Castille pendant que l'entreprise colonisatrice progressait dans les autres îles caraïbes, au Mexique, en Amérique centrale et en Amérique du Sud. Santo Domingo, comme on a vite dénommé le territoire, n'a pas su retenir une forte population blanche. Luttes brutales contre la résistance farouche des autochtones bientôt remplacés pour les besoins de la main-d'oeuvre par des esclaves africains, tremblements de terre et ouragans, déprédations de corsaires, commerce de contrebande actif dans une faible économie axée sur la culture de la canne à sucre... Mise à part l'importance stratégique du lieu, ce n'est pas une bien grande perte que le traité de Ryswick infligeait à l'Espagne en 1697 lorsqu'il la forçait à céder à la France la portion occidentale de l'île. Avant que le traité de Bâle ne lui en accorde aussi la portion orientale, sur laquelle son pouvoir ne pourra d'ailleurs s'exercer vraiment, la France a fait de Saint-Domingue au XVIIIe siècle une riche colonie de plantation dominant la production internationale du café dépendance plus intéressante assurément que les «quelques arpents de neige» laissés à la Grande-Bretagne au nord de l'Amérique en 1763 . Une ambiance de crainte s'est instaurée néanmoins, durant le siècle des Lumières, dans cette possession rentable où la minorité des planteurs blancs redoutait que ne se révoltât la masse des travailleurs noirs et mulâtres. La Révolution française a suscité, entre autres remous, la grande insurrection en 1791 des esclaves de Saint-Domingue. Dans les troubles qui ont alors bouleversé l'île, Toussaint Louverture s'est manifesté comme restaurateur de l'ordre. L'abolition de l'esclavage, votée par la Convention le 4 février 1794, a raffermi la loyauté de ce Noir envers la métropole. Promu général de brigade puis général de division dans l'ar- 
mée française, Toussaint a combattu victorieusement les troupes anglaises et espagnoles, conquérant même au début de 1801 la partie de l'île que l'Espagne a dû promettre à Bâle. Pour le Consulat, cependant, la situation n'était plus très claire à Saint-Domingue. Les «ci-devant Noirs» allaient-ils reconnaître encore l'autorité française et devenir les fidèles citoyens libres de la République une et indivisible? Ou fallait-il plutôt céder aux pressions du parti colonialiste et tâcher de rétablir dans la colonie à pacifier complètement la situation qui prévalait naguère? L'année 1801 fournit au Premier Consul les éléments d'une réponse à la dernière question. Louverture, en effet, promulgue pour Saint-Domingue, le 8 juillet, une constitution le rendant gouverneur à vie et général en chef d'une colonie manifestement autonome. Le traité de Lunéville d'autre part, qui rétablit en février la quiétude avec le monde germanique en attendant que la Paix d'Amiens ne mette fin aux hostilités avec la Grande-Bretagne en mars 1802, atténue pour les armées françaises la tâche guerrière en Europe et permet le départ vers Saint-Domingue d'un corps expéditionnaire de 25000 hommes.

L'histoire de l'Expédition française de Saint-Domingue n'a pas encore été faite de manière scientifique. Nos deux auteurs veulent y contribuer. Leur dessein, qui était d'abord de travailler «sur l'ensemble de la répression» (p. 21), se borne finalement à l'aspect des déportations. Vieille technique de la domination impériale, exercée déjà sur le peuple élu en Babylonie et sur nos Acadiens plus récemment. Au début des généralités qui forment la première partie de l'ouvrage, le plan français de déportation nous est expliqué dans son caractère sélectif, dans la formulation qu'en faisait Napoléon Bonaparte sous le titre de «Notes pour servir aux instructions à donner au Capitaine-général Leclerc», dans sa dimension enfin, et dans sa réalisation. Les «Notes» fixaient au commandant de l'Expédition, qui était donc beau-frère du Premier Consul, le calendrier d'une reconquête totale. Trois étapes, ou «époques», y étaient prévues. La première signifiait l'occupation et l'organisation, la deuxième concernait la poursuite «à outrance» des rebelles, et la troisième ordonnait expressément de «faire arrêter tous les hommes en place suspects, de quelque couleur qu'ils soient et [de] faire embarquer au même instant tous les généraux noirs quels que soient leurs moeurs et leur patriotisme et les services qu'ils ont rendus» (cité pp. 30-31). C'est dans la troisième «époque» que se situe donc le point d'histoire analysé ici. Les gens impliqués sont classés en trois catégories: les «Politiques», ayant participé à l'effort de réorganisation de Louverture et par l'exil desquels le Premier Consul "voulait briser l'épine dorsale de la colonie» (p. 33); les «Militaires», comprenant les cadres de l'armée coloniale que la métropole voulait neutraliser; et les «Sympathisants» qui, sans avoir trahi la France, n'avaient pas su collaborer suffisamment à la campagne de soumission. Combien de personnes au juste ont-elles été arrêtées à travers la colonie en même temps que Louverture était saisi aux Gonaïves le 7 juin 1802? «Aucun décompte officiel n'existe» (p. 38). Écartant les «milliers d'individus» qu'évoquait Jacques Destrem, de même que les 2000 estimés par le général Leclerc parce qu'ils provenaient aussi de la Guadeloupe, les auteurs examinent les listes de passagers des navires uti- 
lisés et arrivent au total de 640 déportés. Ce chiffre «doit seulement représenter une partie de la vérité» (p. 41), mais il ne correspond pas non plus à l'exécution intégrale des «Notes» puisque la troisième «époque» déclenche le soulèvement généralisé qui aboutit à la reddition des troupes françaises le 19 novembre 1803 . Les déportés sont répartis en divers lieux dont la Corse, l'île d'Elbe, Montpellier, Rochefort, Bordeaux, le fort de Joux, le château d'If, Toulon, Paris, Mantoue et Naples. Ils sont emprisonnés, envoyés au bagne ou dans un camp de travail, placés en résidence surveillée, mis à la réforme ou incorporés plutôt au Bataillon Royal Africain que les combats en Italie décimeront complètement. La deuxième partie du livre nous fournit enfin «quelques portraits de déportés» (pp. 82-134) incluant Toussaint Louverture et sa famille, André Rigaud, Martial Besse, Gabriel David Troy ainsi que Jean-Joseph Laplume.

L'objectif du petit ouvrage était «de reconstituer le drame vécu par les indigènes de Saint-Domingue victimes des déportations ordonnées par Bonaparte» (p. 135). Les auteurs ont raison en bonne partie de conclure qu'ils ont bien atteint cet objectif, leur recherche s'appuyant sur un examen patient - sinon exhaustif bien sûr - du thème aux Archives Nationales de France, leurs jugements manifestant une évidente honnêteté intellectuelle, et le texte qu'ils nous proposent gagnant à la sobriété du style. On déplorera peut-être le manque de hardiesse de l'approche conceptuelle. Effort d'histoire patriotique avant tout, nous semble-t-il, cette publication du moins ne pourra être ignorée dans les études futures des origines d'Haïti. 